\title{
Quercetin: Silent Retarder of Fatty Acid Oxidation in Breast Cancer Metastasis Through Steering of Mitochondrial CPT1
}

Bhuban Ruidas ( $\sim$ bhubo18@gmail.com )

Indian Institute of Engineering Science and Technology https://orcid.org/0000-0003-2062-9159

Tapas Kumar Sur

RG Kar Medical College and Hospital: RG Kar Medical College

Chitrangada Das Mukhopadhyay

IIEST Shibpur: Indian Institute of Engineering Science and Technology

Koel Sinha

IIEST Shibpur: Indian Institute of Engineering Science and Technology

Sutapa Som Chaudhury

IIEST Shibpur: Indian Institute of Engineering Science and Technology

Pramita Sharma

The University of Burdwan

Sovonlal Bhowmick

University of Calcutta

Achintya Saha

University of Calcutta

Rabindranath Majumder

IIEST Shibpur: Indian Institute of Engineering Science and Technology

\section{Research Article}

Keywords: Fatty acid oxidation, CPT1, Quercetin, Glyco and Mito-stress, Molecular docking

Posted Date: September 24th, 2021

DOI: https://doi.org/10.21203/rs.3.rs-849234/v1

License: (1) (i) This work is licensed under a Creative Commons Attribution 4.0 International License.

Read Full License 


\section{Abstract}

Recent evidence concreted that maximum energy in metastatic breast cancer progression is supplied by fatty acid oxidation (FAO) governed by a rate-limiting enzyme, carnitine palmitoyltransferase 1 (CPT1). Therefore, active limitation of FAO could be an emerging aspect to inhibit breast cancer progression. Herein, for the first time we have introduced Quercetin (QT) from a non-dietary source (Mikania micrantha Kunth) to seize the FAO in triple-negative breast cancer cells (TNBC) through an active targeting of CPT1. Apart from successive molecular quantification, QT has resulted a significant reduction in the intracellular mitochondrial respiration and glycolytic function limiting extensive ATP production. In turn, QT has elevated the reactive oxygen species (ROS) and depleted antioxidant level to induce anti-metastatic and cell apoptosis activities. Real-time quantitative reverse transcription-polymerase chain reaction (qRT-PCR) investigated the FAO associated gene expression resulting significant depletion in FAO which were further confirmed through the successful in-silico molecular docking prediction for active binding potentiality of QT to CPT1. Subsequently, QT has shown an excellent in-vivo antitumor activities through the altered lipid profile and oxidative stress healing capabilities in female breast cancer BALB/c mice model. Therefore, all the obtained data significantly grounded the fact that QT could be a promising metabolismtargeted breast cancer therapeutics.

\section{Introduction}

Breast cancer is one of the major cancers with a high mortality rate in women across the world. Based on diagnostic evidence, more than $15-20 \%$ of the total breast cancer depicted triple-negative breast cancer (TNBC) with high recurrence and worse survival rate compared to non-TNBC [1-3]. TNBC is a fatal breast cancer subtype with absence of three primary receptors, namely estrogen receptor (ER), progesterone receptor (PR) and human epidermal growth factor receptor 2 (HER-2) [4-6]. This attitude is directly correlated with elevated lipolysis or fatty acid oxidation (FAO) for high energy compensation during rapid proliferation, even in hypoxic conditions [7-9]. The metastatic cancer cell undergoes metabolic rearrangements, including alterations in fatty acid transport, accumulation of lipid droplets, de novo lipogenesis and $\beta$-oxidation to achieve extensive ATP demand $[10,11]$. It has been hypothesized that glycolysis solely plays a significant role in cancer progression. Later, the fact was at stake since the discovery of direct mitochondrial relation with fleeting cancer progression [12, 13]. The mitochondrial bioenergetics and FAO association have been witnessed to stage an imperative role in cancer stemness, survival, proliferation and chemoresistance [14, 15].

Carnitine palmitoyltransferase system acts as a pivotal mediator in cancer metabolic plasticity in active association with FAO. This system is solely responsible for long-chain fatty acid (LCFA) delivery from the cytoplasm to mitochondria for fatty acid $\beta$-oxidation, and carnitine palmitoyltransferase 1 (CPT1) catalyzes rate-limiting steps of FAO. Thereby, CPT1 and FAO targeting breast cancer have been hypothesized to be an optimistic approach towards anticancer therapeutics [16-18]. Besides, fatty acid synthase (FASN), a rate-limiting enzyme, plays a decisive role in LCFA processing in FAO. Overexpression in FASN has been considered to promote breast cancer progression, while up-regulation in acetyl-CoA 
carboxylase (ACC) has shown negative feedback limiting breast cancer regression $[19,20]$. Interestingly, the direct involvement of different enzymes and carriers in the FAO pathway, modulating beta-oxidation in cancer cells, is of intense interest since time immemorial. Moreover, the inhibition exerted by them remains associated with antitumor activities and thereby needs quick surveillance over time.

Despite the availability of several synthetic anticancer drugs, natural drugs have gained the immense spotlight in recent times due to minimal side effects [21]. The natural phytochemicals from edible plants have shown remarkable possibilities towards effective anticancerous treatment with beneficial clinical advantages [22, 23]. Mikania micrantha Kunth (bitter vine or mile-a-minute vine), an invasive weed of the family Asteraceae, contains various bioactive compounds such as flavonoids, phenols, and polyphenols. This globally famed antiseptic and folk medicine are applied for assessment of antibacterial activities mostly and rarely were explored in cancer therapies [24, 25]. Quercetin (QT) [IUPAC name: 2-(3, 4dihydroxyphenyl)-3, 5, 7-trihydroxy-4H-chrome-4-one; CAS number: 117-39-5 6151-25-3], a flavonol with anti-lipid oxidative property has been widely used in cancer treatment. It undergoes various activities like decreases the FASN expression, inhibits proliferation of carcinoma cells and significantly improves the plasma non-enzymatic antioxidant capacity during chemotherapy $[25,26]$. It also elevates the lipid peroxides level, reduces tumor size and the cumulative number of papilloma, and acts as a potent inhibitor of lipogenesis in prostate and breast cancer cells by inhibiting FASN activity $[27,28]$. Reports evidenced that QT potentially inhibit lipid synthesis by suppression of peroxisome proliferator-activated receptor-gamma (PPARY) and CCAAT-enhancer-binding protein a (C/EBPa) and activation of AMPactivated protein kinase (AMPK) in 3T3-L1 cells [29]. Eventually, QT has been proven to modulate the activity of hepatic cholesterol and hepatic cholesterol 7a-hydroxylase, the enzyme involved in cholesterol metabolism, promoting the strategic conversion of cholesterol-to-bile acid [30]. Dietary QT is intriguingly reported to limit FAO in metastatic breast cancer to stimulate DNA damage and apoptosis [31-33]. Therefore, it will be worth exploring QT from non-dietary sources as effective anticancer therapeutics.

In this study, we have synthesized a flavonoid subtype, QT, from Mikania micrantha Kunth and evaluated its metabolism-targeted anticancer activities via checking the outcome of FAO associated rate-limiting enzyme. QT determined the fate of total intracellular glycolysis and mitochondrial oxidation rate promoting cell death and apoptosis. Additionally, overall molecular action has been successfully predicted through in silico molecular binding assay via 3D (three-dimensional) protein structure preparation of CPT1 followed by an extra-precision (XP) molecular docking, elucidating the plausible mechanistic binding interactions of QT with CPT1. Lastly, in-vivo experimentation in female BALB/C breast cancer mice model has portrayed the intracellular lipid profiling and oxidative stress management enabling antitumor activities of QT, confirming its successive metabolism-targeted anticancer activities.

\section{Materials And Methods}

\subsection{Species authentication}


The experimental invasive weed specimen, Mikania micrantha Kunth has been authenticated by K. Karthigeyan, Scientist-'E' of Botanical Survey of India, Botanical Garden, Howrah-711103, West Bengal, India, with species number- PS-01 and the reference number- $\mathrm{CNH} /$ Tech.II/2020/14 dated 18th June 2020.

\subsection{Extract preparation, characterization and estimation of Quercetin}

The invasive plant, Mikania micrantha Kunth was collected from IIEST Shibpur, Howrah, West Bengal, India. After washing and oven-drying at $35^{\circ} \mathrm{C}$ for 30-40 min. Next, Soxhlet extractions were performed followed by spectrometric analysis of the specific compounds (Supporting informations).

\subsection{Cell lines and cytotoxicity assay}

The triple-negative human breast cancer cell lines (MDA-MB-231, MDA-MB-468), normal breast epithelial cell line (MCF-12F) and mice breast cancer cell line (4T1) were purchased from National Centre for Cell Science (NCCS), Pune, Maharashtra, India and were revived according to supplier's protocol (Supporting information). MTT (3-(4,5-dimethylthiazol-2-yl)-2,5-diphenyl tetrazolium bromide) assay checked the cytotoxic effects of QT in MDA-MB-231, MDA-MB-468, MCF-12F and 4T1 cell lines respectively (Supporting information).

\subsection{ROS and Intracellular antioxidant measurements}

2',7' - dichlorofluorescein diacetate (DCFDA) methods were applied to investigate the QT triggered reactive oxygen species (ROS) generation whereas GSH-GloTM Glutathione Assay kit (Promega) and AmpliteTM Fluorometric NADPH Assay (AAT Bioquest, USA) kit found the intracellular GSH and NAPDH level respectively (Supporting information).

\subsection{Intracellular Mito and Glyco-stress analysis}

Seahorse XFe24 Extracellular Flux Analyzer (Seahorse Bioscience, North Billerica, MA, USA) checked the intracellular Mito and Glyco- stress in $24 \mathrm{XF}$ well plates via. The investigation of overall oxygen consumption rate (OCR) and extracellular acidification rate (ECAR) in MDA-MB-231 cells respectively [34]. Detailed experimentation method has been elaborated in supporting informations.

\subsection{Anti-invasion and migration study}

Anti-invasion and migration activities of MDA-MB-231 cells in the presence of QT were investigated using the Transwell assay kit (ECM 555, Millipore) and monolayer cell scratching assay respectively by the invasive and wound healing cell imaging under an inverted fluorescence microscope (Olympus, Japan) and the measurement of relative fluorescence intensities utilizing a microplate reader (Bio Rad, India) following standard protocol (Supporting information).

\subsection{ELISA assay for metastatic protein expression}


Active metastasis related protein, matrix metalloproteinase-2 and 9 (MMP-2 and MMP-9) expression were investigated through protein specific enzyme-linked immunosorbent assay (ELISA) kit (Sigma, Aldrich) following the supplier's protocol (Supporting informations).

\subsection{RNA extraction, cDNA synthesis and gene expression analysis}

Trizol methods were applied for total RNA extraction followed by the preparation of cDNA using normal polymerase chain reaction (PCR, Bio-Rad, Hercules, CA, USA). Then the individual gene expression was achieved through comparative CT method $(\triangle \triangle C T)$ by qRT-PCR (Applied Biosystems, Waltham, MA,USA) after the selection of gene specific forward and reverse primer (IDT technologies, lowa, USA (Table S1) (Supporting information).

\subsection{Cell apoptosis analysis}

Annexin V-FITC and PI double staining method was applied to investigate the apoptotic cell death in MDA-MB-231 cells. The QT-treated cells were washed with chilled 1X PBS buffer before their incubation with $100 \mu$ l of annexin binding buffer. Then the cells were allowed to mix with $5 \mu$ l of Annexin V-FITC (2 $\mu \mathrm{g} / \mathrm{ml})$ and $5 \mu \mathrm{l}$ of $\mathrm{PI}(0.5 \mathrm{mg} / \mathrm{ml})$ and following a $15 \mathrm{~min}$ incubation at room temperature in the dark, the results were recorded via the FACS (BD Biosciences, San Jose, CA).

\subsection{In-silicomolecular modeling and docking studies}

Due to the unavailability of the complete information of 3D crystal structure of CPT1, prediction of 3D structure of CPT1 has been carried out in I-TASSER (Iterative Threading Assembly Refinement) server available at https://zhanglab.ccmb.med.umich.edu/I-TASSER/ followed by the active validation through the Ramachandran plot analysis. [35, 36]. The Glide-XP docking execution was performed employing the 'Ligand docking' module of Schrödinger's Maestro interface following default settings to analyze the CPT1 protein-ligand QT interactions $[37,38]$. As an output parameter, docking result was allowed to generate maximum of 6 docked poses per ligand (Supporting information).

\subsection{Animals}

The nulliparous female BALB/c mice of 6-7 weeks-old with average body weight 20-30 g was used for the maintenance and experiment following $\mathrm{NIH}$ guidelines and institutional animal ethical committee approval (RKC/IAEC/A/03 dated 14/12/17) for laboratory animals at R.G. Kar Medical College, Kolkata, India. A detailed animal care and use were reported earlier [34]. Moreover, a successive in-vivo anticancer assessment including toxicity profiling and therapeutic dosage confirmation were achieved following standard protocols (Supporting information). 


\subsection{Statistical analysis}

Mean \pm standard error of mean (SEM) were preferred to express the experimental data. Multiple comparison tests or one way analysis of variance (ANOVA) and post hoc Dunnett's test software (SPSS v20) analyzed the groups statistically. Also, unpaired 2-tailed student t-test measured the level of significance in some experiments. Statistical significant level was considered with $p$-value of less than 0.001 and 0.05 .

\section{Results}

The spectrophotometric analysis of total flavonoids in MIKA was estimated to $132.66 \mu \mathrm{g}$ equitant to QT for each mg of MIKA. Among the flavonoids, QT was identified and quantified by HPTLC. Densitometric HPTLC was performed accordingly to ICH guidelines for instrumental precision, repeatability, specificity and accuracy. Rf values of marker standard QT was determined as 0.38 and the regression analysis via area of QT was 0.99662 (sdv = 3.29) (Fig. S1). The amount of QT present in MIKA was calculated from the calibration curves and presented in Table $\mathbf{S 2}$.

\subsection{In-vitro anticancer assessment}

\subsubsection{Cell cytotoxicity assay}

From cell cytotoxicity assay, IC $\mathrm{I}_{\mathbf{5 0}}$ value of QT was found to be $30 \mu \mathrm{g} / \mathrm{ml}$ (QT-30) in MDA-MB-231 and 35 $\mu \mathrm{g} / \mathrm{ml}$ (QT-35) in MDA-MB-468 whereas in MCF-12F, it was $85 \mu \mathrm{g} / \mathrm{ml}$ (QT-85) (Fig. S2). Thereby, QT-30 was selected as maximum treatment dose for the experiment.

\subsubsection{Fate of major antioxidant system and ROS}

A high amount of ROS generation is almost a common phenomenon for all cancerous cells while excessive production leads to negative feedback [39]. Herein, almost a $50 \%$ increment in ROS production was investigated with QT-15 treatment while it was almost doubled with QT-30 compared to untreated control (Fig.S3a). This observation indicated that QT triggered ROS mediated anticancer possibilities. The fate of intracellular antioxidant system, mainly GSH and NADPH level are the crucial event to predict the anticancer possibilities actively as a high level of GSH/NADPH are the common phenomena in most of the cancer cells. At QT-45, GSH and NADPH level were dropped around $65 \%$ and $60 \%$ respectively while at QT-30, GSH and NAPDH were dropped almost 55\% and 45\% respectively (Fig. S3b, c).

\subsection{Mitochondrial respiration and total glycolysis}

Intracellular mitochondrial respiration was calculated from total oxygen consumption rate (OCR) comparison (Fig. 1a). In our previous report, we have shown that Palmitate-BSA alone can elevate the intracellular OCR and ECAR rate in breast cancer cells [34]. In this current experiment, intracellular basal and maximal respiration rate were decreased about $23.78 \%$ and $68.69 \%$ respectively in presence of QT-30 compared to control. Also, 35.07\% depletion in maximal respiration was observed in presence of QT-30 
with Palmitate-BSA $(1: 1, \mathrm{v} / \mathrm{v})$ but a slight increase about $17.33 \%$ in basal respiration were also investigated (Fig. 1b, C). Intracellular total proton leak and spare respiratory capacity together exhibit the ATP production. A significant decrement about $62.43 \%$ and $30.92 \%$ in proton leak were found in presence of QT-30 and QT-30 with Palmitate-BSA respectively compared to untreated control. Similarly, $54.33 \%$ and $42.03 \%$ reduction in spare respiratory capacity were also observed in presence of QT-30 and QT-30 with Palmitate-BSA respectively compared to control which in turns indicate the active depletion in intracellular ATP production (Fig. 1d, e).

The glycolytic functions were calculated from total extracellular acidification rate (ECAR) comparison (Fig. 2a). Apart from the mitochondrial respiration alteration, significant reductions in total glycolysis about $77.68 \%$ and $49.16 \%$ were investigated in presence of QT-30 and QT-30 with Palmitate-BSA respectively compared to control. Likewise, glycolytic capacity were also reduced around $94.86 \%$ and $54.28 \%$ in presence of QT alone or with Palmitate-BSA compared to control respectively (Fig. 2b, c). This significant depletion in glycolytic function has further enhanced the QT-triggered energy mediated anticancer possibilities. The molecular mechanism behind these phenomena was further hypothesized comparing relative protein expression profiling related to cancer cell metabolism. In most of the cancer cell, CPT1 expressions are always high due to the continuation of excessive energy supply via fatty acid $\beta$-oxidation. Interestingly, in presence of QT-30 almost a 2 fold and 2.5 fold decrement has been observed in CPT1A and FASN expression respectively whereas, remarkable 3 fold increment was evidenced in ACCa (Fig. 2d). From this observation, it can be concluded that QT-triggered increment in ACCa directly interferes in fatty acid metabolism via down regulation of both CPT1A and FASN.

\subsection{Invasion and migration assay}

An active invasion and migration abilities are the key phenomena in metastatic cancer progression. A dose dependent QT-triggered wound healing ability or cell migration was confirmed through scratch assay analysis. After $24 \mathrm{~h}$ of QT-15 and QT-30 treatment, the wound closure percentages were found about $28 \%$ and $38 \%$ respectively whereas it was about $11 \%$ and $21 \%$ after $48 \mathrm{~h}$ of treatment respectively (Fig. 3a, b). In untreated control, the wound closure percentage was about $78 \%$ after $24 \mathrm{~h}$ and $100 \%$ after $48 \mathrm{~h}$. From Transwell invasion assay, almost 39\% reduction in cell invasion has been observed in presence of QT-15 whereas QT-30 has resulted about 63\% reduction in cell invasion compared to untreated control (Fig. 3c, d). Moreover, MMP-2 level in QT-15 and QT-30 treated cells were found about 54 $\mathrm{pg} / \mathrm{ml}$ and $43 \mathrm{pg} / \mathrm{ml}$ respectively whereas it was about $69 \mathrm{pg} / \mathrm{ml}$ in untreated control. Similarly, MMP-9 level was investigated about $71 \mathrm{pg} / \mathrm{ml}$ in untreated control whereas it was about $57 \mathrm{pg} / \mathrm{ml}$ and $46 \mathrm{pg} / \mathrm{ml}$ in QT-15 and QT-30 treated cells respectively.

\subsection{Cell apoptosis analysis}

A dose dependent apoptotic cell death was observed in presence of QT. In untreated control cells, percentage of apoptotic cells were very low about 0.1 whereas live cells percentage was very high with 99.94 as expected. In QT-15 treated cells, total cell apoptosis and live cell percentage were 15.6 and 34.9 respectively whereas, it was 36.6 and 13.4 in QT-30 treated cells (Fig. S4a, b). Therefore, QT has 
successfully enhanced the live to apoptotic cell transition in breast cancer cells effectively to affirm its anticancer abilities profoundly.

\subsection{In-silico molecular binding interaction analyses of QT to modeled CPT1 protein}

I-TASSER based structural prediction of CPT1 protein revealed that above $90 \%$ of residues in the "core" regions and hence possessed acceptable stereo-chemical quality of the modeled CPT1 protein as illustrated in the Ramachandran plot analysis (Fig. 4a). Moreover, major numbers of amino acid residues are found in most favored regions of the plot which has been validated through the high quality modeled protein.

Moreover, the comprehensive docking analysis has demonstrated that QT formed several numbers of $\mathrm{H}$ bond interactions with amino acid residues Trp236, Tyr241, Ser252, Tyr589 and Thr602 of CPT1 (Fig. 4b). Precisely, several active functional hydroxyl $(-\mathrm{OH})$ groups in QT participated to create $\mathrm{H}$-bond interactions with side-chains residues of CPT1 and the bond distances measured between 1.80 to $2.83 \AA$. In addition, a number of hydrophobic interactions have also been observed with residues Trp236, lle240, Tyr589, Ala591 and Val715 of CPT1. In addition, the binding mode for QT inside the hollow catalytic tunnel of CPT1 has been generated and depicted in Fig. 4c. The surface view orientation of represented binding mode of QT with CPT1 strongly suggests that QT can hold its position tightly inside the hollow catalytic tunnel of $\mathrm{CPT} 1$ at the $\mathrm{COOH}$-terminal. Moreover, the observed multiple $\mathrm{H}$-bond and hydrophobic interactions has also suggested the binding selectivity between QT and CPT1 protein and such intermolecular interactions can influence for exhibiting some degree of biological activity, considering the all-important roles of H-bond and hydrophobic interactions in stabilizing the protein-ligand systems. 3.6 In-vivo anticancer activities analysis

From acute oral toxicity profiling, the dose with $2000 \mathrm{mg} / \mathrm{kg}$ oral administration of QT was selected as non-toxic as well as safe dose for other experiments (Table S3).

An excellent antitumor activity has been observed in presence of QT (Fig. 5a-f). Briefly, the average body weight gain of 4T1 cells-induced mice was $2.7 \mathrm{~g}$ in 3 weeks but the body weight drastically seized by the tumor progression. Remarkably, the body weight gain was restored dose-dependently in presence of QT. QT-15 and QT-30 restored around $21.42 \%$ and $24.96 \%$ of body weight gain respectively whereas positive inhibitor, ETO-30 restored around $33 \%$ of body weight gain compared to untreated tumor control. Likewise, QT-15 reduced $37.01 \%$ and $31.81 \%$ of tumor volume and weight respectively in the breast tissues whereas QT-30 lowered $70.27 \%$ and $61.11 \%$ of tumor volume and weight compared to tumor control. ETO-30 also lowered $72.22 \%$ and $73.19 \%$ of tumor weight and volume respectively. In addition, a time-dependent tumor regression was also investigated. After 21 days of treatment, QT-15 and QT-30 has successfully seized $54.29 \%$ and $70.99 \%$ tumor growth compared to untreated tumor control. Similarly, ETO-30 reduced $75.42 \%$ of tumor growth. Microscopic histopathological evaluations of breast tissues have further supported these findings. In tumor control breast tissue, lumen of the duct was filled with proliferative cells in a disorderly pattern and nuclear basophilicity was noted to be enhanced. QT-15 
treated breast tissue has shown lumen of duct with less proliferative cells in an orderly pattern and nuclear basophilicity was much less than tumor control. In QT-30 and ETO-30 treated breast tissues, the ductal epithelial cells and myoepithelial cells were noted in terminal duct-lobule units surrounded by thick basement membrane along with necrotic lesion in the center of ducts.

Altered oxidative stress and lipidomic profile management has been evidenced to induce apoptosis in breast cancer cell $[40,41]$. The obtained data has shown an excellent decrement in LPO and GSH level whereas a significant increment in SOD level in presence of both QT as well as ETO-30 compared to untreated control has been observed in Fig. 6a. Total LPO in tumor control was about $2.93 \mathrm{nM} \mathrm{MDA} / \mathrm{mg}$ proteinwhereas it has been decreased to 2.47 and $2.04 \mathrm{nM} \mathrm{MDA} / \mathrm{mg}$ protein in presence of QT-15 and QT30 respectively. Likewise, GSH in QT-15 and QT-30 treated tumor tissue was decreased to 5.63 and 4.76 $\mathrm{nM} / \mathrm{mg}$ protein respectively whereas in tumor control, it was $6.06 \mathrm{nM} / \mathrm{mg}$ protein. Also, SOD level in tumor control found around $5.02 \mathrm{U} / \mathrm{mg}$ protein whereas it was increased to 6.08 and $6.85 \mathrm{U} / \mathrm{mg}$ protein in QT-15 and QT-30 treated tumor tissue respectively. In addition, a dose dependent decrement in total cholesterol, LDL, VLDL and triglycerides level were observed in both concentrations of QT and ETO-30 treated tissue compared to untreated tumor control whereas a sharp increase in HDL were also observed in QT and ETO treated tissue samples (Fig. 6b). Respective total cholesterol level in QT-15 and QT-30 treated tissue was 143.5 and $128.83 \mathrm{mg} / \mathrm{dl}$ but in untreated tumor control, it was $158.67 \mathrm{mg} / \mathrm{dl}$. Also, LDL and VLDL in QT-30 treated tissue were found about 72.33 and $16.31 \mathrm{mg} / \mathrm{dl}$ respectively while in QT-15 treated tissue, this respective level was witnessed to about 82.05 and $20.33 \mathrm{mg} / \mathrm{dl}$ compared to 98.04 and $25.67 \mathrm{mg} / \mathrm{dl}$ in untreated tumor control respectively. Total HDL in tumor control was calculated about $26.83 \mathrm{mg} / \mathrm{dl}$ whereas it has been increased to 32.17 and $40.67 \mathrm{mg} / \mathrm{dl}$ in QT-15 and QT-30 treated tissue respectively. FAO associated triglyceride levels were also found to be decreased to 99.83 and $81 \mathrm{mg} / \mathrm{dl}$ respectively in QT-15 and QT-30 treated tissue compared to untreated tumor control with $123.83 \mathrm{mg} / \mathrm{dl}$ of triglycerides (Fig. 6c). Thereby, these results have indicated that QT has an active control over these major oxidative stress and lipid parameter to promote antitumor activities or apoptotic cell death.Perhaps, the liver enzymes (SGOT, SGPT and ALP) and renal functions (urea and creatinine) in the serum remains unchanged compared to QT-15, QT-30, ETO-30 and mock treated mice signifying the advantages of QT as a potent anticancer drug (Fig. S5).

\section{Discussion}

FAO is the key phenomenon to promote metabolic plasticity in metastatic cancer cells starting from cytoplasm to mitochondria $[15,42,43]$. In accordance to the above fact, active limitation of FAO in metastatic TNBC could be a novel anticancer approach. Surprisingly, plant-based flavonoids derivative, QT has shown noteworthy activities over available anticancer drugs due to its non-toxic effects along with high efficacy and potentiality [44]. Moreover, QT has been judiciously envisaged to be the most promising anticancer agent but needs further investigation for its establishment as an au courant anticancer therapeutics $[45,46]$. 
Herein, QT has shown a remarkable CPT1 targeted anticancer activities with an excellent control over the intracellular glycolysis, mitochondrial respiration and FAO. It has successfully up-regulated ACCa to inhibit the FASN and CPT1 expression seizing the mitochondrial $\beta$-oxidation which has been further confirmed through the significant depletion in mitochondrial respiration and overall ATP production along with reduction in glycolytic functions limiting extensive energy requirements of TNBC. Moreover, mitochondrial respiration has been influenced by the changes in mitochondrial membrane potential and excessive ROS generation regulating the intracellular antioxidant system which in turn, exalted the antimetastatic activities of QT followed by the induction of programmed cell death or apoptosis. This also

promotes antitumor activity via the management of major in-vivo oxidative stress and altered lipid profile associated with metastatic cancer progression. These activities have been further justified through the insilico molecular binding potentiality of QT to CPT1.

In conclusion, QT has successfully targeted FAO both in vitro and in vivo inhibiting extensive energy supply to promote successive TNBC growth inhibition and could be adjudged as current age anticancer therapeutics.

\section{Abbreviations}

3D- Three-dimensional

ACC-Acetyl-CoA carboxylase

AMPK- Activated protein kinase

ATP-Adenosine triphosphate

C/EBPa- CCAAT-enhancer-binding protein a

CPT1- Carnitinepalmitoyltransferase 1

DCF- Dichlorofluorescein

DCFDA-2',7' -dichlorofluorescein diacetate

ELISA-Enzyme-linked immunosorbent assay

ER- Estrogen receptor

ETC-Electron transport chain

FAO- Fatty acid oxidation

FASN- Fatty acid synthase

FFA-Free fatty acid 
HBSS- Hanks balanced salt solution

HER-2- Human epidermal growth factor receptor 2

LCFA- Long chain fatty acid

MMP- Matrix metalloproteinase

PPARY- Peroxisome proliferator-activated receptor-gamma

PR-Progesterone receptor

qRT-PCR- Real time quantitative reverse transcription polymerase chain reaction

QT- Quercetin

TCA-Tricarboxylic acid

TMB- 3,3',5,5'-tetramethylbenzidine

TNBC- Triple negative breast cancer

XP- Extra-precision

\section{Declarations}

\section{Authors' contributions}

Conceptualization and design of study: BR. Experimentation, Data acquisition and analysis: BR, PS, SSC, SB, TKS. Drafting manuscript: BR, KS. Critical revision of the manuscript: CDM, AS. Study supervision: BR. All the author have read and approved the final manuscript.

\section{Funding}

This work was not supported by any grant from funding agencies.

\section{Conflict of interest}

The authors declare that they have no competing interests.

\section{Ethical approval}

All procedures performed involving animals were in accordance of $\mathrm{NIH}$ guidelines and institutional animal ethical committee approval (RKC/IAEC/A/03 dated 14/12/17) for laboratory animals at R.G. Kar Medical College, Kolkata, India. 
Not applicable.

\section{References}

1. K. Anders, V. Abramson, T. Tan, R. Dent, The evolution of triple-negative breast cancer: from biology to novel therapeutics. Am.Soc.Clin. Oncol. Educ. Book. 35, 34-42 (2016)

2. M. Perou, T. Sorlie, M.B. Eisen, M.van de Rijn, S. S. Jeffrey, C. A. Rees, J. R. Pollack, D. T. Ross, H. Johnsen, L. A. Akslen, O.Fluge, A.Pergamenschikov, C. Williams, S. X. Zhu, P. E. Lonning, A.L. Borresen-Dale, P. O. Brown, D. Botstein, Molecular portraits of human breasttumours. Nature406, 747$752(2000)$

3. Gonçalves, M.R. Guerra, J.R. Duarte Cintra, V.A. Fayer, I.V. Brum, M.T. Bustamante Teixeira, Survival study of triple-negative and non-triple-negative breast cancer in a Brazilian cohort. Clin. Med. Insights Oncol. (2018).doi:10.1177/1179554918790563

4. Tomao, A. Papa, E. Zaccarelli, L. Rossi, D. Caruso, M. Minozzi, P. Vici, L. Frati, S. Tomao, Triplenegative breast cancer: new perspectives for targeted therapies. Onco. Targets Ther. 8, 177-193 (2015)

5. The cancer genome atlas network, Comprehensive molecular portraits of human breast tumours. Nature 490, 61-70 (2012)

6. R. Bauer, M. Brown, R.D. Cress, C.A. Parise, V.Caggiano, Descriptive analysis of estrogen receptor (er)negative, progesterone receptor (pr)-negative, and her2-negative invasive breast cancer, the so-called triple-negative phenotype: a population-based study from the California cancer registry. Cancer 109,1721-1728 (2007)

7. Zhang, G. Du, Dysregulated lipid metabolism in cancer. World J Biol. Chem.3, 167-174 (2012)

8. Koundouros, G.Poulogiannis, Reprogramming of fatty acid metabolism in cancer. British $\mathrm{J}$ Cancer $122,4-22(2020)$

9. Ma, S.M. Temkin, A.M. Hawkridge, C. Guo, W. Wang, X.Y. Wang, X. Fang, Fatty acid oxidation: an emerging facet of metabolic transformation in cancer. Cancer Lett. 435, 92-100 (2018)

10. E. Monaco, Fatty acid metabolism in breast cancer subtypes. Oncotarget, 8, 29487-29500 (2017)

11. K. Nomura, J. Z. Long, S. Niessen, H. S. Hoover, S. W. Ng, B. F. Cravatt,Monoacylglycerol lipase regulates a fatty acid network that promotes cancer pathogenesis. Cell, 140, 49-61(2010)

12. Warburg, F. Wind, E. Negelein,Themetabolism of tumors in the body. J Gen. Physiol. 8, 519-530 (1927)

13. E. Porporato, N. Filigheddu, J. N. V. S. Pedro, G. Kroemer, L. Galluzzi, Mitochondrial metabolism and cancer. Cell Research,28, 265-280 (2018)

14. Wang, J. F. Fahrmann, H.Y. Lee, J. Li, S. C. Tripathi, C. Yue, C. Zhang, V. Lifshitz, J. Song, Y. Yuan, G. Somlo, R. Jandial, D. Ann, S. Hanash, R. Jove, H. Yu, JAK/STAT3-regulated fatty acid b-oxidation is critical for breast cancer stem cell self-renewal and chemoresistance. Cell Metab. 27, 136-150 e5(2018) 
15. Carracedo, L. C. Cantley, P. P.Pandolfi, Cancer metabolism: fatty acid oxidation in the limelight. Nat Rev Cancer. 13, 227-232 (2013)

16. A. BMelone, A. Valentino, S. Margarucci, U. Galderisi, A. Giordano, G. Peluso, The carnitine system and cancer metabolic plasticity. Cell Death and Dis. 9, 228 (2018)

17. Camarda, A.Y. Zhou, R. A. Kohnz, S. Balakrishnan, C. Mahieu, B. Anderton, H. Eyob, S. Kajimura, A. Tward, G. Krings, D. K. Nomura, A.Goga, Inhibition of fatty acid oxidation as a therapy for MYCoverexpressing triple-negative breast cancer. Nat. Med. 22, 427-432 (2016)

18. Qu, F. Zeng, X. Liu, Q. J. Wang, F. Deng, Fatty acid oxidation and carnitine palmitoyltransferase I: emerging therapeutic targets in cancer. Cell Death and Disease. 7, e2226 (2016)

19. A. Hunt, H. M. Lane, M. E. Zygmont, P. A. Dervan, R. A. Hennigar, mRNA stability and over expression of fatty acid synthase in human breast cancer cell lines. Anticancer Res. 27, 27-34(2007)

20. Chajes, M. Cambot, K. Moreau, G. M. Lenoir, V. Joulin, Acetyl-coA carboxylase $a$ is essential to breast cancer cell survival. Cancer Res. 66 (2006)

21. A. Ashraf, Phytochemicals as potential anticancer drugs: time to ponder nature's bounty. BioMed. Res. Int.2020, 8602879 (2020)

22. R. Guerra, F. Maria, M. F. Duarte, I. F. Duarte, Targeting tumor metabolism with plant-derived natural products: emerging trends in cancer therapy. J Agric Food Chem. 66, 10663-10685 (2018)

23. C. Koh, C. T. Ho, M. H. Pan, Recent advances in cancer chemoprevention with phytochemicals. J Food and Drug Analysis 28, 14-37 (2020)

24. M. Sheam, Z. Haque, Z. Nain, Towards the antimicrobial, therapeutic and invasive properties of Mikania micrantha Kunth: a brief overview. J AdvBiotechnolExpTher. 3, 92-101(2020)

25. Khatun, L. Nasrin, S. Roy, M. A. Tantry, M. A.Abdur Rahman, Comparative antimicrobial evaluation of available Mikania species in Bangladesh. Int J Plant Res. 7, 36-38 (2017)

26. Daker, S. Bhuvanendran, M. Ahmad, K. Takada, A.S.Khoo, Deregulation of lipid metabolism pathway genes in nasopharyngeal carcinoma cells. Mol Med. Rep. 7, 731-741 (2013)

27. Tabaczar, A. Pieniążek, J. Czepas, J. PiaseckaZelga, K. Gwoździński, A.Koceva-Chyła,Quercetin attenuates oxidative stress in the blood plasma of rats bearing DMBA-induced mammary cancer and treated with a combination of doxorubicin and docetaxel. Gen PhysiolBiophys. 32, 535-543 (2013)

28. Ali, S. Dixit,Quercetin attenuates the development of 7, 12-dimethyl benz (a) anthracene (DMBA) and croton oil-induced skin cancer in mice. J Biomed Res. 29, 139-144 (2015)

29. Ahn, H. Lee, S. Kim, J. Park, T. Ha, The anti-obesity effect of quercetin is mediated by the AMPK and MAPK signaling pathways. BiochemBiophys Res Commun. 373, 545-549 (2008)

30. Brusselmans, R.Vrolix, G. Verhoeven, J.V. Swinnen, Induction of cancer cell apoptosis by flavonoids is associated with their ability to inhibit fatty acid synthase activity. J Biol Chem. 280, 5636-5645 (2005)

31. Zhang, Z.Xie, W. Gao, L. Pu, J. Wei, C. Gao, Quercetin regulates hepatic cholesterol metabolism by promoting cholesterol-to-bile acid conversion and cholesterol efflux in rats. Nutr Res.36, 271-279 
(2016)

32. S. Sultan, M. I. M. Khalil, B. M. Sami, A. F. Alkhuriji, O.Sadek,Quercetin induces apoptosis in triplenegative breast cancer cells via inhibiting fatty acid synthase and beta-catenin. Int. J. Clin. Exp. Pathol. 10, 156-172 (2017)

33. Zhao, J. M. Mao, S. Y. Zhang, Z. Q. Zhou, Y. Tan, Y. Zhang, Quercetin induces HepG2 cell apoptosis by inhibiting fatty acid biosynthesis. Oncol. Lett. 8, 765-769 (2014)

34. Ruidas, T. K. Sur, K. Pal, S. S. Chaudhury, P.Prasad, K. Sinha, P.K. Sarkar, P. Das, C. D. Mukhopadhyay, Herbometallic nano-drug inducing metastatic growth inhibition in breast cancer through intracellular energy depletion. Mol. Biol. Rep.47, 3745-3763 (2020)

35. Yang, R. Yan, A. Roy, D. Xu, J. Poisson, Y. Zhang, The I-TASSER suite: protein structure and function prediction. Nature meth. 12, 7-8 (2015)

36. Consortium TU: uniProt: a worldwide hub of protein knowledge. Nucl.Acids Res. 47, D506-D515 (2018)

37. A. Friesner, R. B. Murphy, M. P. Repasky, L. L. Frye, J. R. Greenwood, T. A. Halgren, P. C. Sanschagrin, D. T. Mainz, Extra precision glide: docking and scoring incorporating a model of hydrophobic enclosure for protein-ligand complexes. J Med. Chem. 49, 6177-6196 (2006)

38. Salentin, S. Schreiber, V. J. Haupt, M. F. Adasme, M. Schroeder, PLIP: fully automated protein-ligand interaction profiler. Nucleic Acids Res. 43, W443-W447 (2015)

39. Ruidas, S. S. Chaudhury, K. Pal, P. K. Sarkar, C. D. Mukhopadhyay, A novel herbometallic nanodrug has the potential for antibacterial and anticancer activity through oxidative damage. Nanomedicine, 14, 1173-1189 (2019)

40. Vera-Ramirez, P. Sanchez-Rovira, M. C. Ramirez-Tortosa, C. L. Ramirez-Tortosa, S. Granados-Principal, J. A. Lorente, J. L. Quiles, Free radicals in breast carcinogenesis, breast cancer progression and cancer stem cells; biological bases to develop oxidative-based therapies. Crit Rev Oncol Hematol. 80, 347-368 (2011)

41. Llaverias, C. Danilo, I. Mercier, K. Daumer, F. Capozza, T.M. Williams, F. Sotgia, M. P. Lisanti, P. G. Frank,Role of cholesterol in the development and progression of breast cancer. Am J Pathol. 178, 402-412 (2011)

42. Medes, A. Thomas, S. Weinhouse, Metabolism of neoplastic tissue. IV. A study of lipid synthesis in neoplastic tissue slices in vitro. Cancer Res. 13, 27-29 (1953)

43. Montesdeoca,M. Lopez, X. Ariza, L. Herrero, K.Makowski, Inhibitors of lipogenic enzymes as a potential therapy against cancer. FASEB J 34, 11355-11381 (2020)

44. V. Anand David, R. Arulmoli, S. Parasuraman, Overviews of biological importance of quercetin: a bioactive flavonoid. Pharmacogn Rev.10, 84-89 (2016)

45. Srivastava,R. Somasagara, M. Hegde, M. Nishana, S. K. Tadi, M. Srivastava, B. Choudhary, S. C. Raghavan, Quercetin, a natural flavonoid interacts with dna, arrests cell cycle and causes tumor regression by activating mitochondrial pathway of apoptosis. Sci. Rep. 6, 24049 (2016) 
46. Reyes-Farias, C. Carrasco-Pozo, The anti-cancer effect of qt: molecular implications in cancer metabolism. Int J Mol Sci. 20, 3177 (2019)

\section{Figures}

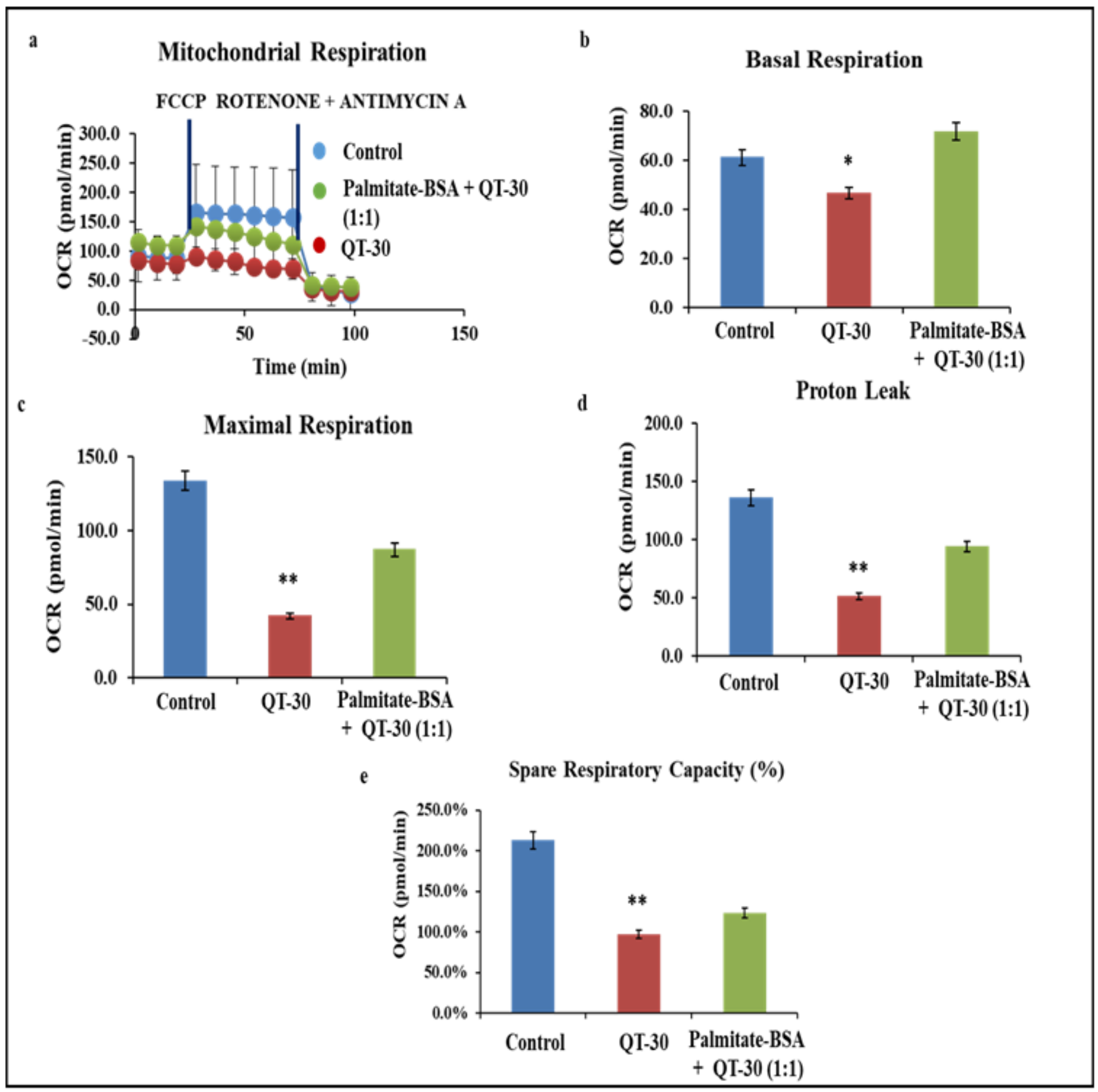

Figure 1 
Fate of Mitochondrial respiration in presence Quercetin (a) the fate of overall mitochondrial oxidation has been depicted viathe illustration of oxygen consumption rate (OCR) in presence of QT-30 and QT-30with fatty acid precursor, palmitate-BSA (1:1) compared to untreated control. A sharpdepletion in basal mitochondrial respiration (b)mitochondrial maximal respiration (c), proton leak (d) and spare respiratory capacity (e) has been illustrated in presence of QT-30 and QT-30 with palmitate-BSA in comparison to untreated control. Bar reports mean \pm s.e.m, one of two experiments completed with or without human subject samples, is shown. ${ }^{\star} p<0.05,{ }^{* *} p<0.001$.

a

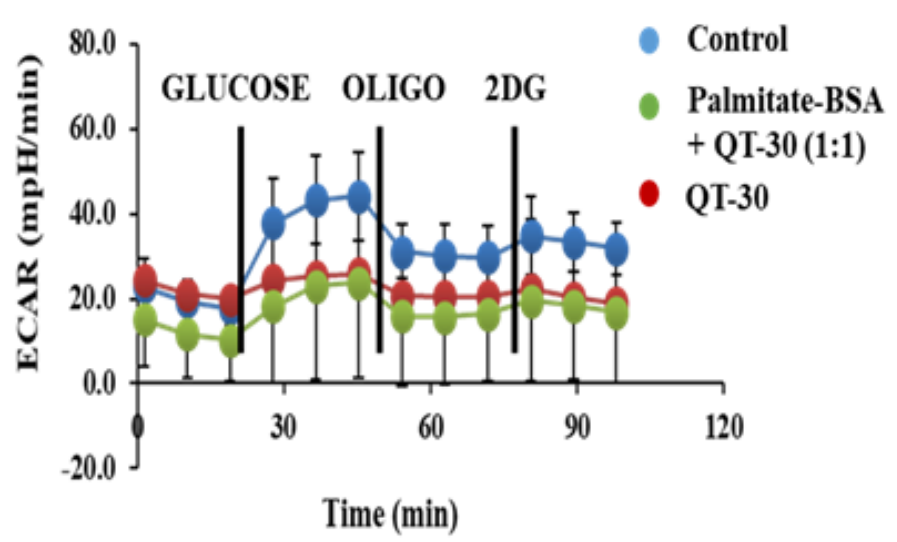

c

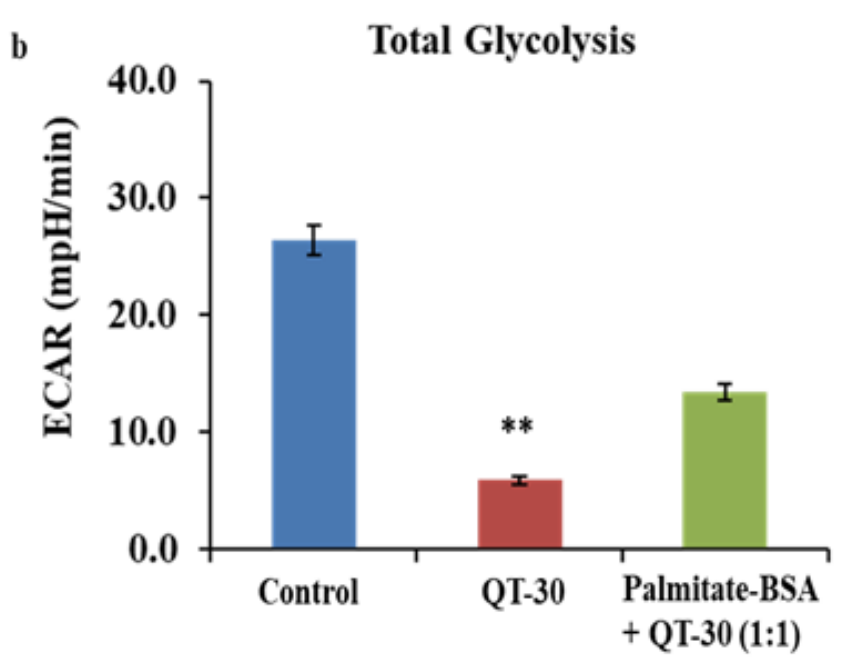

d

Relative gene expression analysis

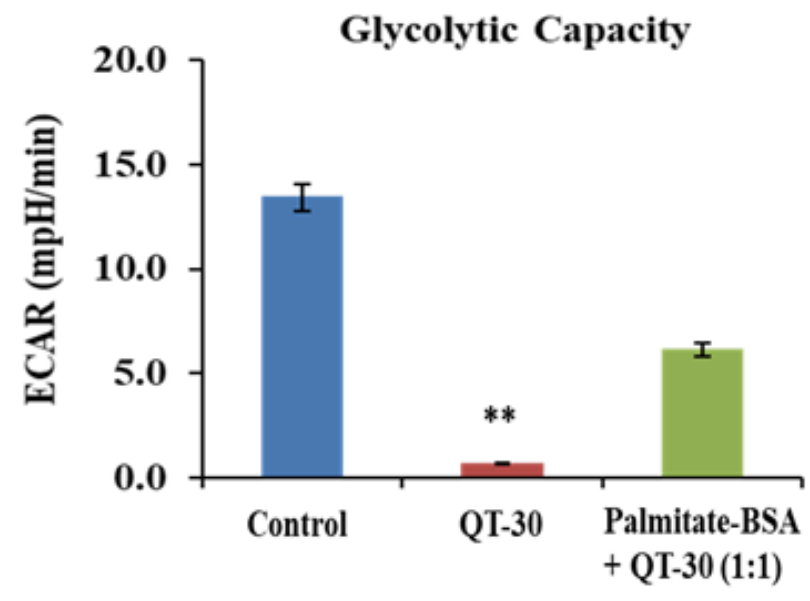

$\square$ Control $\square$ PA-BSA $\square$ PA-BSA+ QT-30 $\square$ QT-30

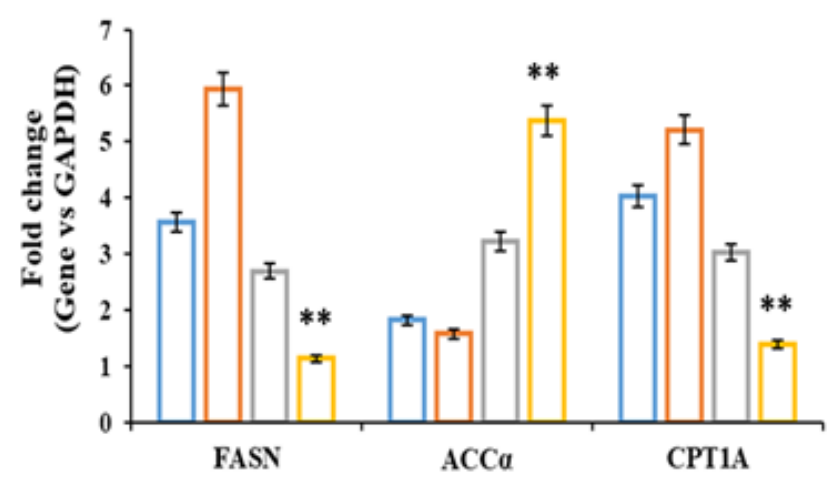

\section{Figure 2}

Fate of Glycolysis and relative FAO associated gene expression The glycolytic function has been illustrated based on the overall extracellular acidification rate (ECAR) in presence of QT-30 and QT-30 with fatty acid precursor, palmitate-BSA (1:1) compared to untreated control (a). A sharp reduction intotal Glycolysis (b) and Glycolytic capacity (c) were represented in presence of QT-30 and QT-30 with 
palmitate-BSA in comparison to untreated control. (d) Changes in the expression of FAO associated major rate limiting enzymes i.e. FASN, ACCa and CPT1A were represented in presence of QT-30 with or without palmitate-BSA along with untreated control. Bar reports mean \pm s.e.m, one of two experiments completed with or without human subject samples, is shown. ${ }^{* *} \mathrm{p}<0.001$.

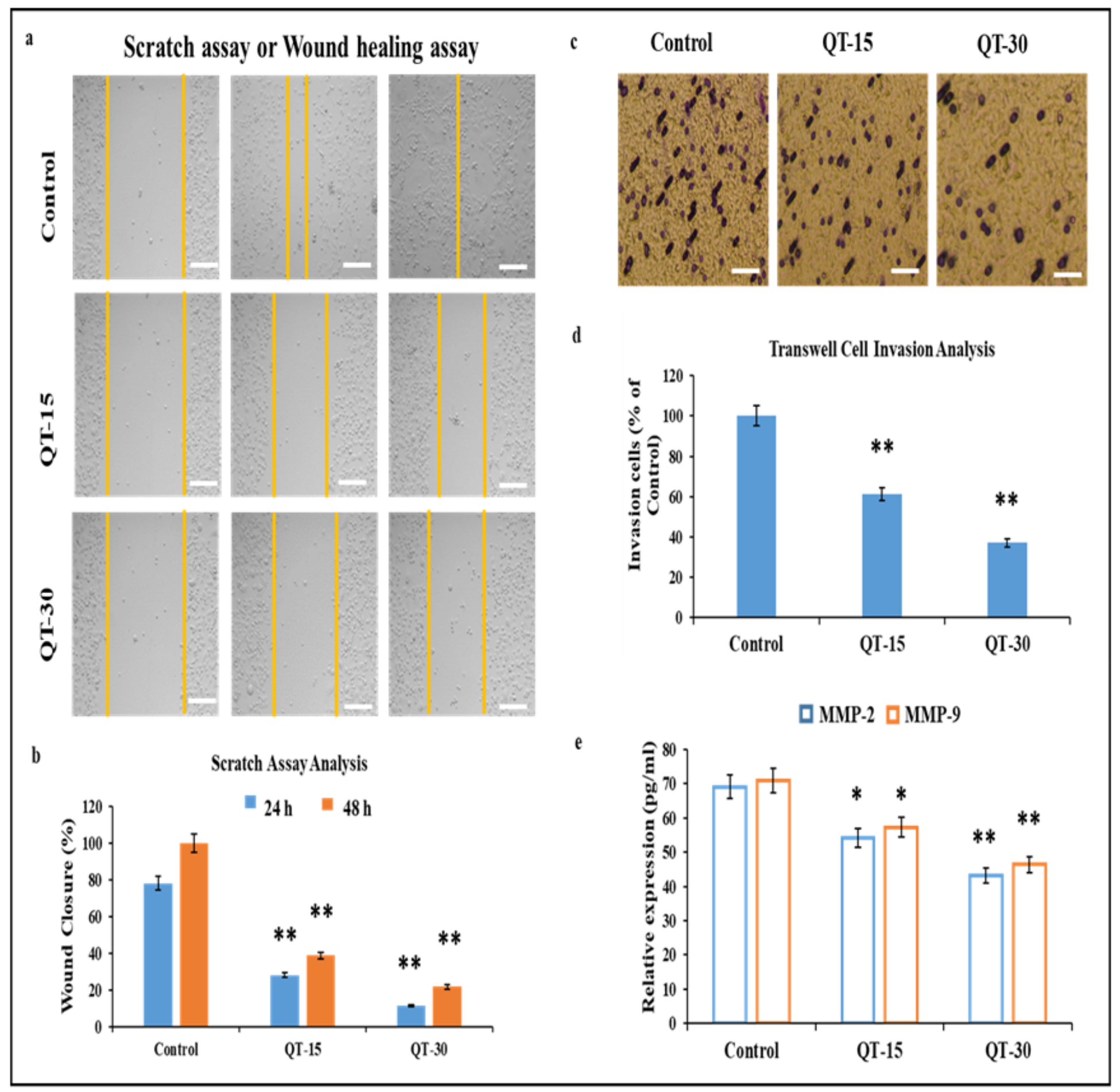

Figure 3

Anti-invasion and anti-migration analysis with relative protein expression QT-triggered successive antimigration of MDA-MB-231 cells were depicted by wound healing assay (a) followed by the investigation 
of quantitative measurements of wound closure percentages after $24 \mathrm{~h}$ and $48 \mathrm{~h}$ of incubation respectively in presence ofQT-15 and QT-30 compared to untreated control (b). Transwellcell invasion assay (c) has shown the QT-triggered anti-invasion abilities whereas the comparative cell invasion has been quantified simultaneously in presence of QT-15 and QT-30 in comparison to untreated control (d). A significant changes in cell migration and invasion related proteins expression i.e. MMP 2 and MMP 9 were representedin presence ofQT-15 and QT-30 compared to untreated control respectively. Bar reports mean \pm s.e.m, one of two experiments completed with or without human subject samples, is shown. ${ }^{*} \mathrm{p}<0.05,{ }^{* *} \mathrm{p}<0.001$.

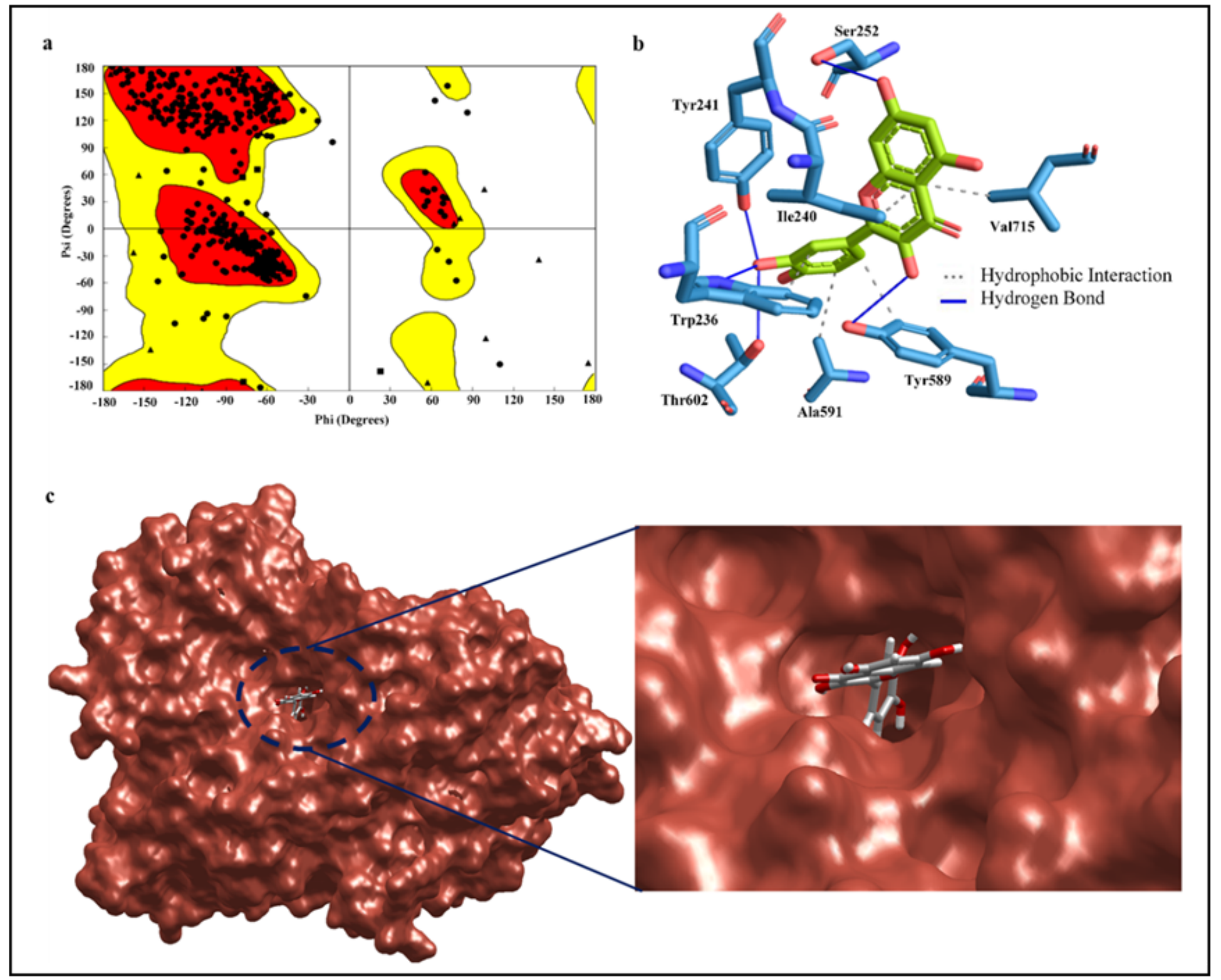

\section{Figure 4}

In-silico molecular docking analysis. (a) The Ramachandran plot shows phi-psi torsion angles for all residues of the modeled CPT1 crystal structure. Glycine residues are indicated by triangles as those are not restricted to the regions of the plot appropriate to the other side-chain types. The darkest areas in red 
correspond to the "core" regions representing the most favorable amino acids of CPT1 and their combinations of phi-psi values. (b) Intermolecular interactions profile between QT and CPT1 obtained in Glide-XP molecular docking analysis. (c) Close surface view representation of the Glide-XP docking based binding mode of QT in the active site of human CPT1 modeled protein

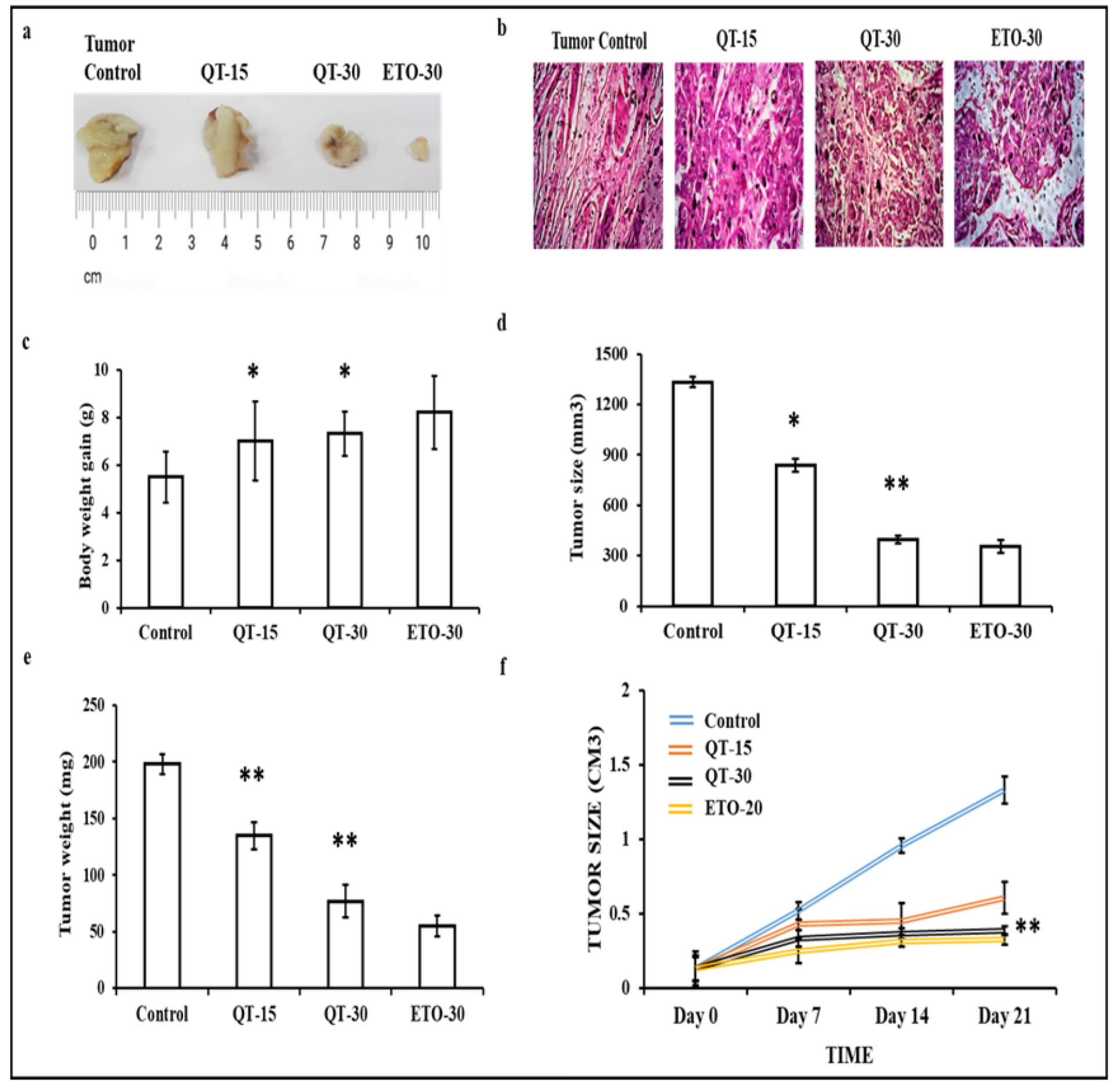

Figure 5

Quantification of in-vivo tumor regression. (a) QT-15, QT-30 and ETO-30 triggered successive tumor regression has been shown compared to tumor control. The comparative changes in tissue histochemistry (b), relative body weight gain (c), comparative tumor size (d) and tumor weight (e) were 
represented in presence of QT-15, QT-30 and ETO-30 compared to untreated tumor control. A timedependent reduction in tumor size has also been depicted in presence of QT-15, QT-30 and ETO-30 compared to tumor control (f). Bar reports mean \pm s.e.m, one of two experiments completed with or without human subject samples, is shown. ${ }^{*} p<0.05,{ }^{* *} p<0.001$.

a

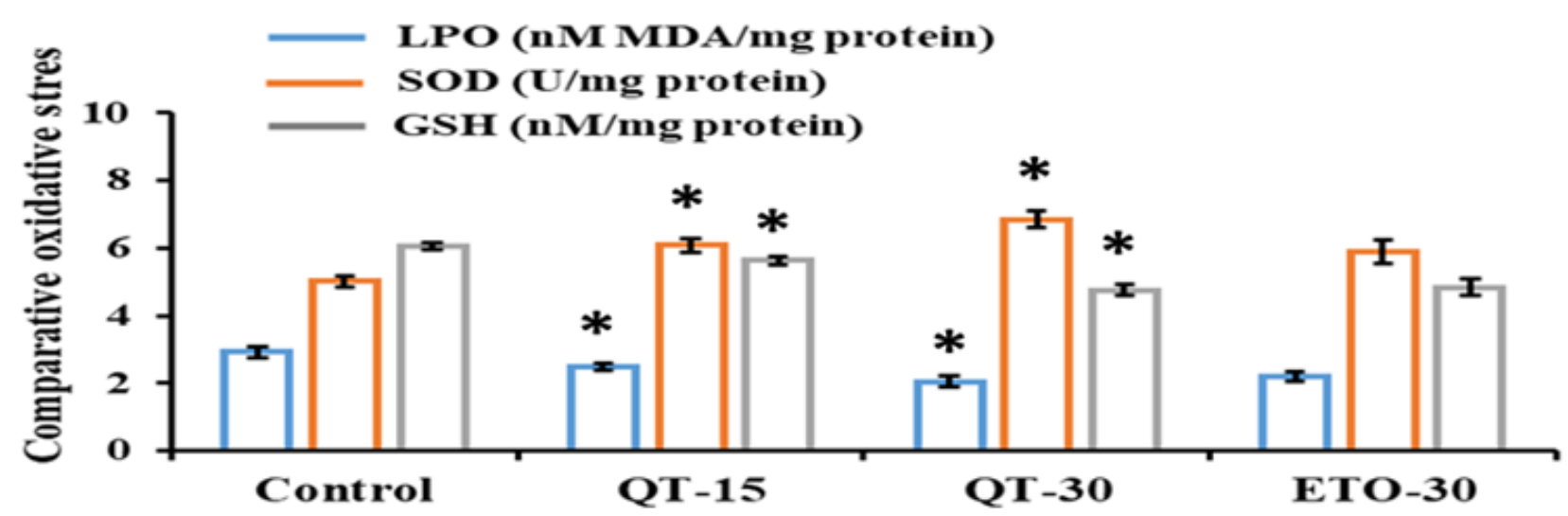

b

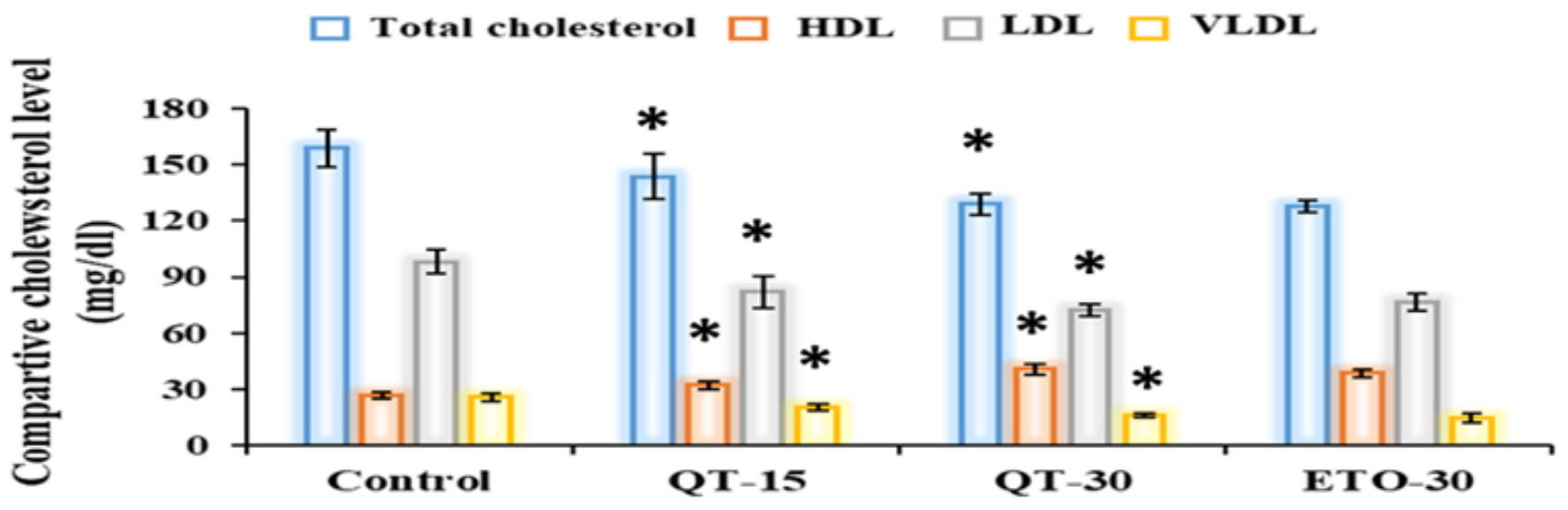

c

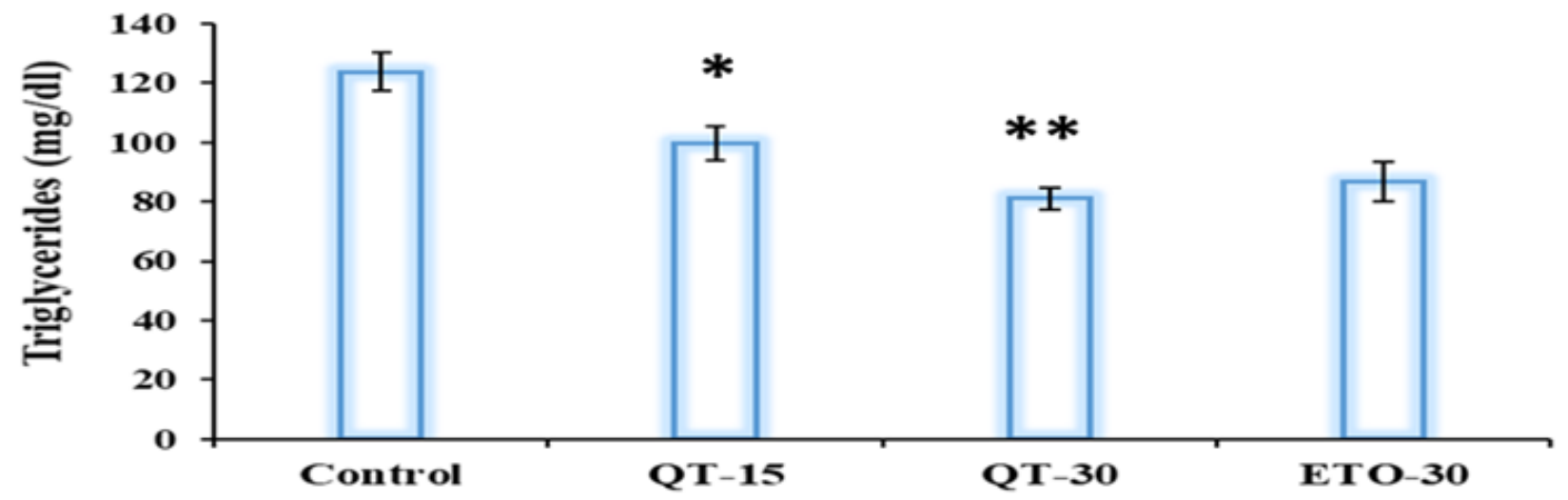

Figure 6

QT-mediated in-vivo oxidative stress and lipid management. The comparative changes in major in-vivo antioxidant level, such as relative LPO, SOD and GSH has been shown in presence of QT-15, QT-30, ETO30 and tumor control (a). A significant increment in total cholesterol, LDL and VLDL followed by the 
increment in HDL has been depicted in presence of QT-15, QT-30 and ETO-30 compared to untreated tumor control (b). A sharp depletion in triglycerides level were represented in presence of QT-15, QT-30 and ETO-30 in comparison to untreated tumor control (c). Bar reports mean \pm s.e.m, one of two experiments completed with or without human subject samples, is shown. ${ }^{*} p<0.05,{ }^{* \star} p<0.001$

\section{Supplementary Files}

This is a list of supplementary files associated with this preprint. Click to download.

- Graphicalabstract.png

- Supportinginformation..docx 